\title{
Upper oesophageal gastric heterotopia: a prospective necropsy study in children
}

\author{
S VARIEND, A J HOWAT \\ From the Department of Pathology, Children's Hospital, Sheffield
}

SUMMARY Three hundred specimens of oesophagus obtained at necropsy from infants and children aged from 0 to 14 years, dying from a variety of causes, were examined prospectively for evidence of gastric heterotopia. Gastric heterotopia was observed in $63(21 \%)$ of the whole series, representing a much higher incidence than reported previously. Excluding deaths in the perinatal period, the incidence appeared to be inversely related to age. Heterotopia was restricted to the subcricoid level of the oesophagus and often showed a close association with lymphoid tissue. There was no association with congenital malformation, and heterotopia occurred more commonly in those infants whose deaths were unexplained, although the reason for this association was unclear.

Barrett's oesophagus, a condition in which the gastric mucosa lines the lower oesophagus in continuity with the stomach as a complication of reflux injury to the lower oesophagus, has been described in adults ${ }^{1}$ and children. ${ }^{2}$ Gastric mucosa presenting above the diaphragm may also occur in association with a hiatus hernia, which may itself be associated with reflux and coexist with Barrett's oesophagus. ${ }^{3}$ Gastric mucosa may also present in the upper part of the oesophagus where it appears to represent an example of true heterotopia, in contrast to that presenting at the lower end of the oesophagus. The existence of upper oesophageal gastric mucosa has been recognised for a long time, and the lesion has formed the basis of several reports since the early part of this century.

The first comprehensive account in the English language was by Hewlett, ${ }^{4}$ who found superficial gastric glands in the upper oesophagus in five of 10 cases at necropsy. The description of the lesion was amplified by Schridde, who reported on 30 such cases at necropsy.' He found macroscopic evidence of gastric tissue at this site in $70 \%$ of cases aged 6 months to 68 years. The heterotopic tissue occurred as bilateral oval craters with the greatest diameter being in the longitudinal oesophageal plane. The gastric tissue was restricted to the mucosa and there was no extension deep to the muscularis mucosae.

Taylor subsequently reported six cases of upper oesophageal gastric heterotopia found on gross inspection in 900 cases at necropsy. ${ }^{6}$ This represented an incidence of $0.7 \%$ and there was a male:female ratio of $5: 1$. This contrasted with the incidence of $7 \cdot 8 \%$ reported by Rector and Connerley, who studied 1000

Accepted for publication 3 March 1988 consecutive cases at necropsy with an age range from 0 to 14 years. ${ }^{7}$ That study, however, was retrospective and purely histological, with blocks having been taken at random levels.

Studies on live patients are rarely reported. Adults subjected to endoscopic examination and biopsy of the upper oesophagus showed gastric mucosal islands in 40 of 1000 examinations. $^{8}$

A wide discrepancy exists as to the precise incidence of upper oesophageal gastric heterotopia and this study was undertaken in an attempt to resolve the actual incidence among children. The investigation was prospective and combined macroscopic and histological methods, special attention being paid to the immediate subcricoid level where the lesion is known to be most common.

\section{Material and methods}

Three hundred consecutive necropsies on children with an age range from 0 to 17 years were studied between September 1984 and June 1987. The cause of death varied widely. The reason for the unusually high proportion of unexplained deaths is that this department carries out necropsies on all home deaths throughout south Yorkshire. Necropsies on hospital deaths within this area are usually performed by the respective hospital pathologists, and such cases were not included in this study.

In each case the oesophagus was opened longitudinally in the posterior midline plane and the mucosa scrutinised macroscopically for abnormality. Attention was paid particularly to the region just below the cricoid. After fixation in formalin for about three 
weeks blocks were taken from the immediate subcricoid area, and also from the middle of the middle third, and mid-lower third (fig 1). Transverse blocks were taken specifically to include craters or mucosal irregularity in the subcricoid area, but even when abnormality was not visualised, blocks were also taken from the same area, usually $0.5 \mathrm{~cm}$ to $1 \mathrm{~cm}$ below the level of the cricoid, depending on age and size of the specimen. Sections were stained with haematoxylin and eosin. Macerated stillbirths which showed exfoliation of the oesophageal epithelium on microscopy were excluded.

There were 82 cases in the perinatal age group which included 43 stillbirths (group A), 184 cases between 1 week and 2 years (group B), and 34 older than 2 years (group C). There were 45 males and 37 females in group A, 109 males and 75 females in group B, and 20 males and 14 females in group $C$. In group $B$ there were 112 cases of unexplained infant death and 72 cases in which death was attributable to recognised disease (explained deaths).

Presence of heterotopia was compared with variables such as cause of death, presence of congenital abnormality, age and sex. The size of the lesion and associated microscopic features such as inflammation and presence of parietal cells were also recorded.

Statistical analysis was by $\chi^{2}$ test.

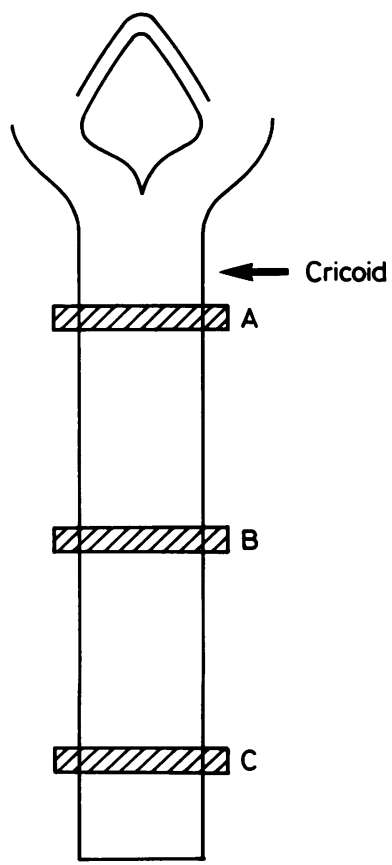

Fig 1 Levels of oesophagus from which tissue was sampled for histological examination.

\section{Results}

For the entire series the incidence of upper oesophageal gastric heterotopia was $62(21 \%)$. The male:female ratio of the whole group was 1.4:1. Most $30(48 \%)$ of the lesions were easily identified by naked eye (fig 2), and some 15 or $25 \%$ were suspected by naked eye and confirmed microscopically. The remainder, 17 or $27 \%$, required microscopy for their detection.

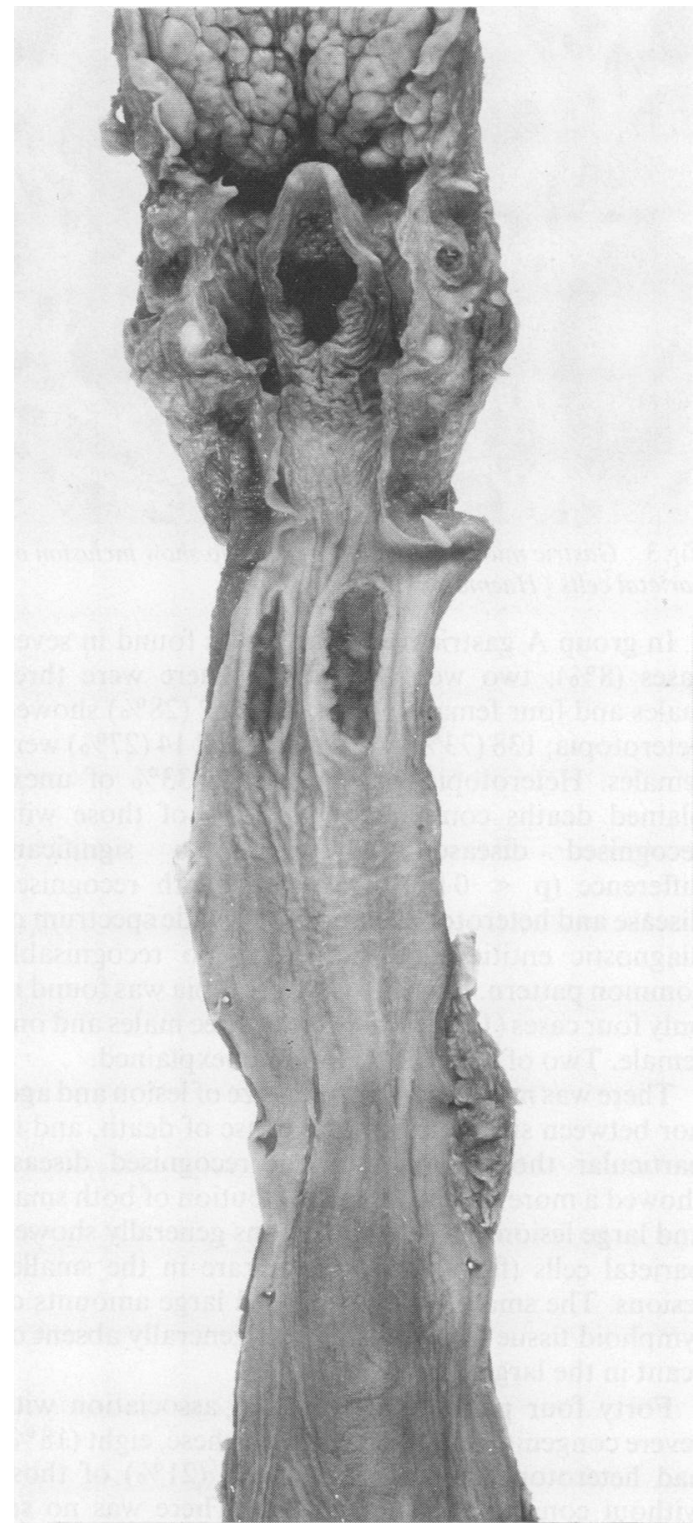

Fig 2 Oesophageal mucosa showing two symmetrical oval craters in subcricoid region. 


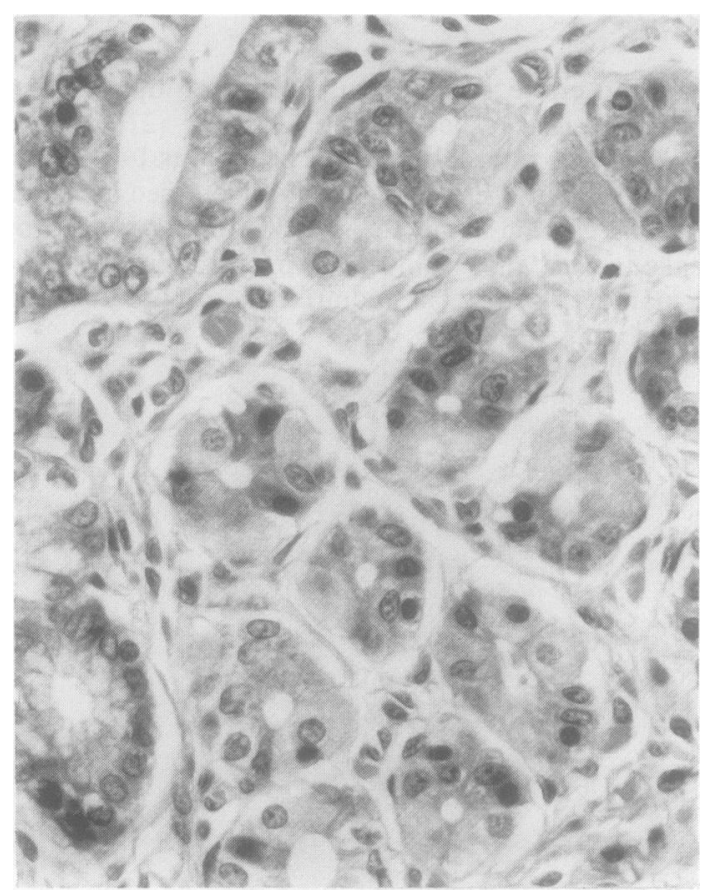

Fig 3 Gastric mucosa from large crater to show inclusion of parietal cells (Haematoxylin and eosin.)

In group A gastric heterotopia was found in seven cases $(8 \%)$; two were stillbirths. There were three males and four females. In group B $52(28 \%)$ showed heterotopia: $138(73 \%)$ were males and $14(27 \%)$ were females. Heterotopia was present in $33 \%$ of unexplained deaths compared with $17 \%$ of those with recognised disease, representing a significant difference $(p<0.02)$. The group with recognised disease and heterotopia comprised a wide spectrum of diagnostic entities and there was no recognisable common pattern. In group $\mathrm{C}$ heterotopia was found in only four cases $(12 \%)$. There were three males and one female. Two of these deaths were unexplained.

There was no relation between size of lesion and age, nor between size of lesion and cause of death, and in particular those dying without recognised disease showed a more or less equal distribution of both small and large lesions. The larger lesions generally showed parietal cells (fig 3) which were rare in the smaller lesions. The small lesions exhibited large amounts of lymphoid tissue (fig 4), which was generally absent or scant in the larger lesions.

Forty four patients had died in association with severe congenital malformation. Of these, eight (18\%) had heterotopia compared with $55(21 \%)$ of those without congenital malformation. There was no set pattern among those with congenital malformation and they comprised Beckwith syndrome $(n=1)$, severe congenital heart disease $(n=2)$, congenital diaphragmatic hernia $(n=2)$, a large laryngeal cleft ( $n$ $=1)$, trisomy $18(n=1)$ and achondrogenesis type 1 $(\mathrm{n}=1)$.

\section{Discussion}

The incidence of upper oesophageal gastric heterotopia in this study was considerably higher than that reported in earlier studies. There are three possible explanations for this difference: first, the lesion is more common in children: secondly, previous studies have been retrospective, with random sampling of tissue for microscopy; thirdly, many previous investigations have relied on naked eye examination, without recourse to histological examination. The incidence cited here may, in fact, be an underestimate as minute lesions in some of the cases may have escaped sectioning and detection.

The exceptionally high incidence during the first year and its apparent decline with age suggests that the lesion regresses with age. The mechanism of the regression is not known but may have an inflammatory basis related to the presence of lymphoid tissue, which was observed in a high proportion of the cases.

An analysis of the data reported by Rector and Connerley reveals a similar decline in incidence with advancing years ${ }^{7}: 10 \%$ of their cases between birth and 7 days showed heterotopia; between 7 days and 12 months there was also an incidence of $10 \%$. During the second year the incidence was $8 \%$ and thereafter it was $4.5 \%$. The higher incidence reported here compared with that given by Rector and Connerley is probably due to random sampling of the oesophagus for microscopy in their series. They estimated the level of origin of the section based on the pattern of musculature in the wall of the oesophagus, a method which they admitted was an approximation. Consequently, many of the sections from the upper third of the oesophagus in their study probably originated from a level lower than where the lesion actually occurred.

The comparatively low incidence of heterotopia in the group between birth and 1 week runs counter to the concept that the incidence is inversely related to age. The data of Rector and Connerley are not too dissimilar to those presented here in this respect as they found an incidence of $10 \%$ before 1 week as well as between the age of 1 week and 12 months, and a subsequent decrease in incidence. There is no immediate explanation for this observation.

Heterotopia had a significantly greater incidence among those dying inexplicably and the reason for this is also unclear. The lesion has been causally related to sudden death in one infant, ${ }^{9}$ allegedly producing massive inhalation of gastric content. Tracheo-bronchial inhalation was not an uncommon finding in our series but occurred more or less equally in those with and without oesophageal heterotopia. There were certainly many cases with heterotopia but not associated with post mortem evidence of gastric inhalation.

The precise genesis of upper oesophageal heterotopia is unknown. Heterotopia, however, is 


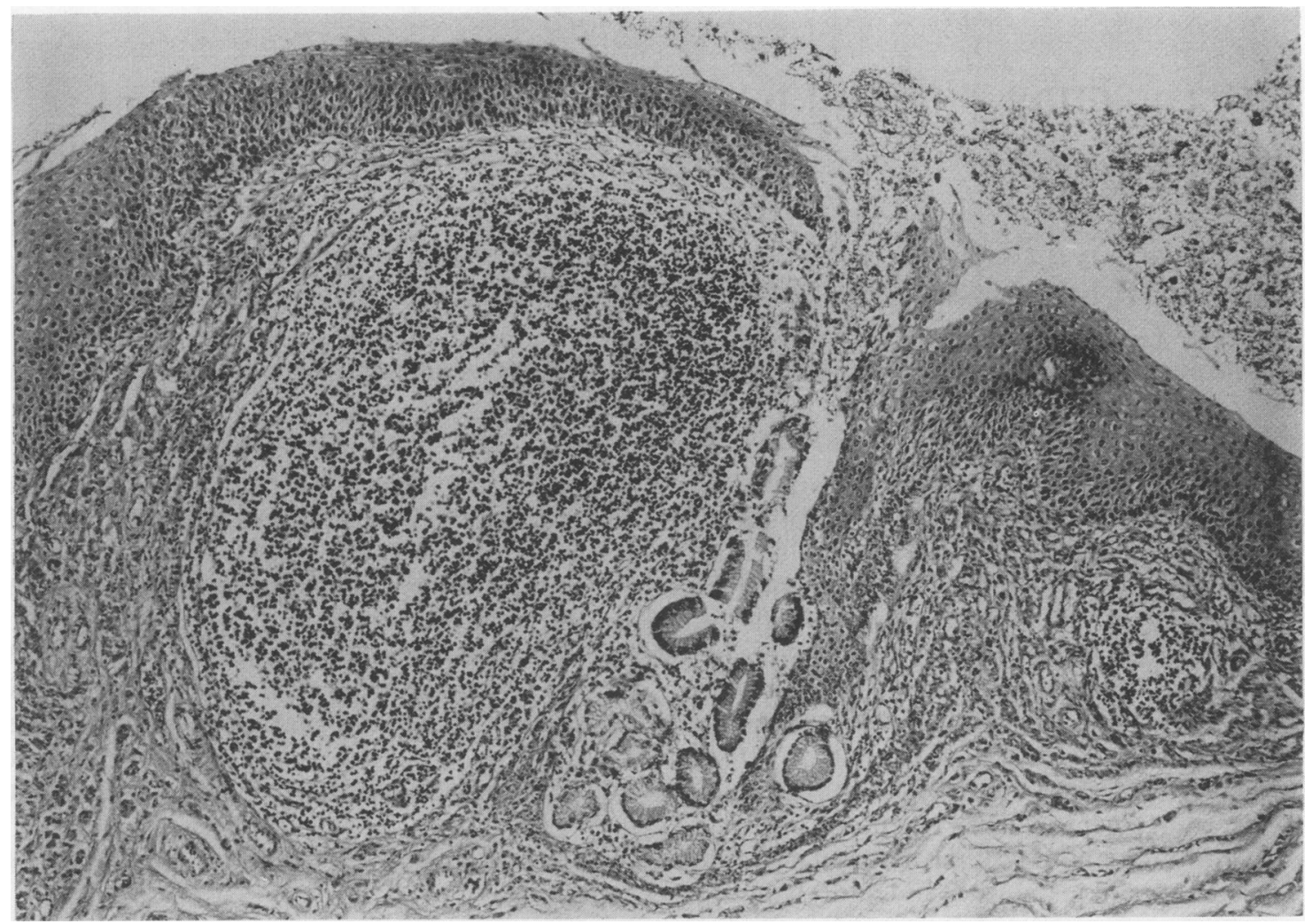

Fig 4 Small heterotopic lesion shows cardiac type gastric glands and prominent lymphoid tissue (Haematoxylin and eosin.)

located in an area of preceding complex embryonic activity. It has been suggested that this may result in failure of cells to differentiate in this area. ${ }^{4}$ Alternatively, the high position of the embryonic stomach in the neck and its subsequent descent may lead to mucosal rests remaining in the upper oesophagus.?

In $34 \%$ of infants with oesophageal atresia, gastric mucosa was observed at the caudal end of the proximal pouch ${ }^{10}$ and in that study $2.5 \%$ of 400 control infants showed heterotopia at a comparable level. Rector and Connerley occasionally also found the lesion in the middle third. ${ }^{7}$ In our study gastric heteropia was limited to the immediate subcricoid region and was not found at other oesophageal levels. The discrepancy is difficult to explain.

While upper oesophageal gastric heterotopia has been regarded as asymptomatic, ${ }^{6}$ it has been linked with dysphagia in an adult ${ }^{11}$ and oesophageal stricture in close proximity to gastric heterotopia at this level has been reported in an elderly woman. ${ }^{12}$

\section{References}

1 Allison PR, Johnstone AS. The oesophagus lined with gastric mucous membrane. Thorax 1953;8:87-101.

2 Dahms BB, Rothstein FC. Barrett's esophagus in children: a consequence of chronic gastroesophageal reflux. Gastroenterology 1984;86:318-23.
3 Cooper JE, Spitz L, Wilkins BM. Barrett's oesophagus in children: a histological and histochemical study of 11 cases. $J$ Pediatr Surg 1987;22:191-6.

4 Hewlett AW. The superficial glands of the oesophagus. J Exp Med 1901;5:319-31.

5 Schridde $H$. Uber magenschleimhaut-Inseln vom bau der cardialdrusenzone und fundusdrusenregion und den unteren, oesophagealen cardialdrusen gleichende drusen im obersten oesophagusabschnitt. Virchows Arch (Pathol Anat) 1904;175:1.

6 Taylor AL. The epithelial heterotopias of the alimentary tract. $J$ Pathol Bacteriol 927;30:415-49.

7 Rector LE, Connerley Marion L. Aberrant mucosa in the esophagus in infants and in children. Archives of Pathology 1941;31:285-94.

8 Ottenlann R, Kunert H, Kuhner W, Seib HJ. MagenschleimhautInseln im zervikalen osophagus. Dtsch Med Wochenschr 1983;108:246-9.

9 Libcke JH. Heterotopic gastric mucosa in the cervical esophagus a possible cause of fatal aspiration. Pediatrics 969;44:447-8.

10 Emery JL, Haddadin AJ. Gastric-type epithelium in the upper oesophageal pouch in children with tracheo-oesophageal fistula, J Pediatr Surg 1971;6:449-53.

11 Foxen EHM. Ectopic gastric mucosa in the cervical oesophagus, a possible cause of dysphagia. J Laryng Otol 1957;71:419-23.

12 Weaver GA. Upper esophageal web due to a ring formed by à squamocolumnar junction with ectopic gastric mucosa (another explanation of the Paterson-Kelly, Plummer-Vinson syndrome). Dig Dis Sci 1979;24:959-3.

Requests for reprints to: Dr S Variend, Department of Histopathology, The Children's Hospital, Western Bank, Sheffield S10 2TH, England. 